\title{
Dynamic formation of oriented patches in chondrocyte cell cultures
}

\author{
Marcus J. Grote · Viviana Palumberi • \\ Barbara Wagner • Andrea Barbero • Ivan Martin
}

Received: 20 July 2010 / Revised: 23 November 2010 / Published online: 14 December 2010

(C) Springer-Verlag 2010

\begin{abstract}
Growth factors have a significant impact not only on the growth dynamics but also on the phenotype of chondrocytes (Barbero et al. in J. Cell. Phys. 204:830$838,2005)$. In particular, as chondrocytes approach confluence, the cells tend to align and form coherent patches. Starting from a mathematical model for fibroblast populations at equilibrium (Mogilner et al. in Physica D 89:346-367, 1996), a dynamic continuum model with logistic growth is developed. Both linear stability analysis and numerical solutions of the time-dependent nonlinear integro-partial differential equation are used to identify the key parameters that lead to pattern formation in the model. The numerical results are compared quantitatively to experimental data by extracting statistical information on orientation, density and patch size through Gabor filters.
\end{abstract}

Keywords Cell alignment $\cdot$ Pattern formation $\cdot$ Stability $\cdot$ Integro-partial differential equations · Image processing

Mathematics Subject Classification (2000) $\quad 92 \mathrm{C} 17 \cdot 92 \mathrm{D} 25 \cdot 92-08 \cdot 65 \mathrm{M} 06$

\footnotetext{
M. J. Grote $(\varangle) \cdot$ V. Palumberi

Institute of Mathematics, Rheinsprung 21, 4051 Basel, Switzerland

e-mail: Marcus.Grote@unibas.ch

B. Wagner

Weierstrass Institute for Applied Analysis and Stochastics, Mohrenstr. 39, 10117 Berlin, Germany
}

A. Barbero - I. Martin

Department of Biomedicine, University Hospital, Hebelstrase 20,

4031 Basel, Switzerland 


\section{Introduction}

In recent years, therapies for damaged tissue have experienced great progress through the possibilities offered by new methods of tissue engineering (Langer and Vacanti 1993). In particular, this emerging field holds great promise for the regeneration of tissues with limited intrinsic repair capacity like adult articular cartilage. Most procedures pursued in tissue engineering start with a biopsy containing few cells, which are expanded ex vivo making use of cytokines. Those are then injected into a patient to grow tissue in vivo. Alternatively, scaffolds are used to provide mechanical support and structure for the tissue to be grown in vitro before its implantation on the defect. Those procedures may involve tissue replacement using donor tissue or autologous cells for in vitro cell-culture expansion, in order to regenerate tissue that matches the patient's native tissue.

Much research has already gone into the impact of combinations of growth factors on the proliferative capacity for a range of cell types, such as pancreatic endocrine cells, neural progenitor cells, muscle-derived stem cells (Beattie et al. 1997; Carpenter et al. 1999; Stewart et al. 2003; Deasy et al. 2002) and chondrocytes (Jakob et al. 2001; Barbero et al. 2003, 2004, 2005). For muscle-derived stem cells and chondrocyte cell cultures, mathematical models that establish characteristic kinetic parameters, such as the fraction of dividing cells and mean cell division-time have been developed (Deasy et al. 2002; Barbero et al. 2005). In addition, a logistic delay-differential model for proliferating chondrocyte cells was used to further include the effect of contact inhibition of proliferating cells upon confluence (Barbero et al. 2005).

Apart from the impact of growth factors on kinetic parameters, an important focus of research concerns the effect of cytokines on the phenotype of individual cells and the resulting organizational structure of the cell culture. Both will influence the mechanical properties of the engineered tissue, which in the case of cartilage, is intended to sustain tensile stresses and compressive loads, just as native tissue does (Sharma and Elisseeff 2004). Therefore, it is important to understand the underlying processes leading to the formation of large-scale patterns of an evolving cell culture. Identifying the relevant parameters that control these structures is the focus of the present study, combining experimental and mathematical methods (Chipot and Edelstein-Keshet 1983).

In Barbero et al. (2005), adult human articular chondrocytes (AHAC) were isolated from cartilage biopsies and then cultured in the presence of a combination of growth factors. The individual cells assume a phenotype that closely resembles fibroblasts and eventually self-organize into regions of aligned cells, making up the monolayer of the cell culture at confluence (Barbero et al. 2003). This phenomenon has been observed before for various cell types. In principle there are a number of mechanisms that may control the formation of such patterns, ranging from chemical, adhesive or other mechanical gradients, see, e.g. Trinkaus (1985) for an early but instructive discussion in the context of morphogenesis.

Even in the absence of exterior influences, however, Elsdale and Bard (1972) discovered that proliferating fetal lung fibroblasts form parallel arrays during in vitro cell expansion. Similar results were found for BHK fibroblasts in the experimental study by Erickson (1978). Elsdale and Bard (1972) argued that the intrinsic property of fibroblasts is to move, unless prevented to do so by the environment, and 
hence that patterns form solely due to direct cell-cell interactions to enable maximal motility. Under the assumption of contact inhibition, Erickson (1978) concluded from a series of cell-cell contact experiments that if the lamellipodium of a cell in ruffling mode contacts another cell at a certain angle, the direction of motion changes depending on that part of the leading edge of the lamellipodium which made contact and where ruffling is stopped. This mechanism is employed by Erickson to explain the existence of a critical angle above which cells cease to align. This critical angle seems to differ for different cell types, e.g. about $20^{\circ}$ for fetal lung fibroblasts and approximately $50^{\circ}$ for BHK cells. For fibroblasts the leading edge of the lamellipodium is much narrower than for the BHK fibroblasts. Hence, except for rather narrow contact events, motion will halt (else cells may even criss-cross other cells). Moreover, similar behaviour is observed for contact events of already established arrays of aligned cells. This behaviour is eventually reflected in the resulting patterns at confluence.

Mathematical modeling of the dynamical process of array formation of aligned cells started with the work by Edelstein-Keshet and Ermentrout (1990). The continuum models derived for pattern forming cell cultures assume random spatial and orientational distributions of the cells that are attracted (repulsed) and change their direction of motion in response to cell-cell interactions. Here the cell density depends on time, two-dimensional physical space and the angle of orientation. The range of interaction is kept small in order to model the local character of cellular interactions. Apart from terms modeling the random motion in physical and angular space, the model includes a term that describes the probability of alignment of cells as a response to cell-cell contact, which vanishes outside the range of angles known to lead to alignment. In subsequent articles the resulting system of integro-differential equations for free cells and cells already bound to an array are discussed in various limiting cases and analysed with respect to their stability about the homogeneous state (Mogilner and Edelstein-Keshet 1995). Similar models were also used for other pattern forming processes such as swarming or the dynamics of actin binding fibers (Mogilner and Edelstein-Keshet 1995; Mogilner et al. 1996; Civelecoglu and Edelstein-Keshet 1994).

Here we extend these models by including time-dependent logistic growth to account for the later stages of in vitro chondrocyte cell expansions. In fact, one important aspect of our study is to enable a direct comparison with our experimental results in Sect. 2.1. The analysis of the experimental results and, in particular, the classification of the cells within angular space is realized by using two-dimensional Gabor filters (Daugman 1985) for the experimental images and is described in Sect. 2.3. In Sect. 3, we present our mathematical model, which consists of a time-dependent nonlinear integro-partial differential equation. We use standard finite differences for the numerical discretization in space, for the time discretization we use explicit Chebyshev methods that circumvent the crippling stability restrictions of standard explicit Runge-Kutta methods-see Appendix. In Sect. 4, we investigate the stability of the solution via a linear stability analysis about the homogeneous state and compare those findings to the results of the full nonlinear model. Finally, quantitative comparisons with experimental data are performed in Sect. 5 . 


\section{Biological background}

\subsection{The impact of growth factors}

Depending on the cell type and the specific growth factors used, cytokine-induced proliferation of cells can generally be characterized by one or more parameters, such as a shorter cell division time, a shorter time until first cell division, or lower percentage of remaining quiescent cells (Deenick et al. 2003; Deasy et al. 2002; Barbero et al. 2005). Those key parameters can be obtained, for instance, by combining a logistic delaydifferential model with the results from specific micro-colony experiments (Barbero et al. 2005). From that model, Barbero et al. established in the case of adult human articular chondrocytes (AHAC) expansion in a medium supplemented with the growth factor combination TGF $\beta-1$, FGF-2 and PDGF-BB (TFP) that the time of first cell division is about 1.4 times shorter and the percentage of quiescent cells about 1.7 times smaller than in the absence of TFP.

Further characteristics observed in experiments (Barbero et al. 2005) concern the elongated shape the cells assume when cultured in a medium with TFP. During the sigmoidal growth of the cell culture, individual cells are initially oriented at random. As the population approaches confluence, cells tend to locally align and form coherent structures. Those spatial patterns appear highly irregular while individual patches greatly vary both in shape and size, without clear boundaries between them (see Fig. 1).

\subsection{Cell culture: isolation and expansion}

To monitor patch formation and obtain quantitative experimental data on diffusion constants, we track the motion of an ensemble of individual AHAC cells up to confluence. Here full-thickness human articular cartilage samples were collected from the femoral lateral condoyle of two individuals (patient A: male, 18 years old, patient B:
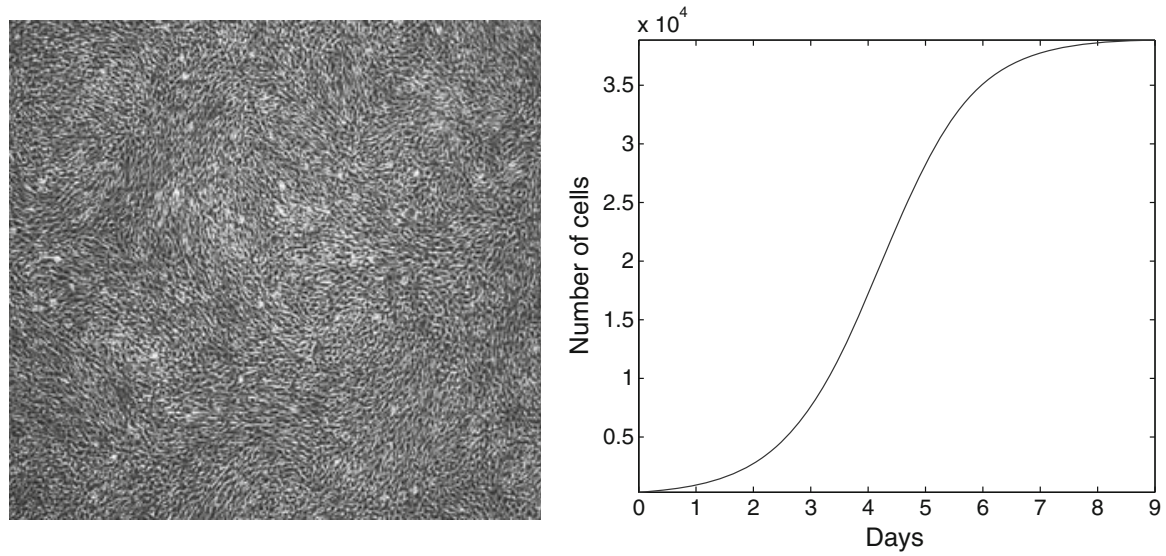

Fig. 1 AHAC cultured with TFP at confluence, day 9 (left). Sigmoidal evolution of the number of cells versus time (right) 
male, 66 years old), with no history and no radiographic signs of joint disease, after informed consent and in accordance with the local Ethical Commission. Adult human articular chondrocytes (AHAC) were isolated using $0.15 \%$ type II collagenase for $22 \mathrm{~h}$ and cultured for one passage in Dulbeccos modified Eagles medium (DMEM) containing $10 \%$ foetal bovine serum, $4.5 \mathrm{mg} / \mathrm{ml}$ D-glucose, $0.1 \mathrm{mM}$ nonessential amino acids, $1 \mathrm{mM}$ sodium pyruvate, $100 \mathrm{mM}$ HEPES buffer, $100 \mathrm{U} / \mathrm{ml}$ penicillin, $100 \mu \mathrm{g} / \mathrm{ml}$ streptomycin, and $0.29 \mathrm{mg} / \mathrm{ml} \mathrm{L-glutamine}$ and supplemented with the $1 \mathrm{ng} / \mathrm{ml}$ of Transforming Growth Factor-b1 (TGF-b1), $5 \mathrm{ng} / \mathrm{ml}$ of Fibroblast Growth Factor-2 (FGF-2) and $10 \mathrm{ng} / \mathrm{ml}$ of Platelet-Derived Growth Factor-BB (PDGF-BB) (growth factor medium, TFP) in a humidified $37^{\circ} \mathrm{C} / 5 \% \mathrm{CO}_{2}$ incubator as previously described (Barbero et al. 2005). When cells were approximately $80 \%$ confluent, first passage (P1) cells were rinsed with phosphate buffered saline, detached using $0.05 \%$ trypsin/0.53 mM EDTA and frozen in complete medium containing $10 \%$ dimethylsulfoxid. AHAC after thawing were then used for the studies described below.

\section{Monitoring of cell expansion until confluence}

AHAC were re-plated in two wells of a 6 well plate at a density of 10,000 cells $/ \mathrm{cm}^{2}$ and cultured in growth factor medium up to 10 days in a humidified $37^{\circ} \mathrm{C} / 5 \% \mathrm{CO}_{2}$ incubator with daily culture medium change. AHAC cultures were monitored by phase contrast microscopy and pictures were taken from random areas of the wells each day.

\section{Study of cell movement}

AHAC were re-plated in a six-well plate at different densities, which were 200, 3,000, $10,000,15,000$ and 20,000 cells $/ \mathrm{cm}^{2}$, and cultured in growth factor medium for 1 day in a humidified $37^{\circ} \mathrm{C} / 5 \% \mathrm{CO}_{2}$ incubator. Next, the plate was transferred to the incubator of the Olympus system. From a time-lapse microscope we obtained a sequence of frames which we used to monitor cell motion. Snapshots were taken at $15 \mathrm{~min}$ intervals, which corresponds to an average travel distance of $9 \mu \mathrm{m}$, to provide sufficient space-time resolution compared to the typical length of a cell $(50 \mu \mathrm{m})$. With the software analySIS ${ }^{D}$ we performed a manual tracking in all five wells (Fig. 2) to follow about 100 cells for each density, for $12 \mathrm{~h}$, a duration that allowed us to neglect cell doubling.

\section{Spatial diffusion}

To estimate the spatial diffusion, we performed experiments at various densities (i.e. 200, 3,000, 10,000, 15,000, 20,000 cells/scm) and for each density we manually tracked individual cells in three different areas of the well. Assuming Brownian motion, the diffusion coefficient $D$ is related to the mean square displacement, $\left\langle X^{2}\right\rangle$, through the relation $\left\langle X^{2}\right\rangle=2 D t$. A linear least-squares fit of the time evolution of the mean square distance then yields $D$. From those estimates at varying density, shown in Table 1, we obtained the constant average diffusion coefficient $D=0.29 \mu \mathrm{m}^{2} / \mathrm{s}$. 
Fig. 2 Tracking of individual cells at density $20,000 \mathrm{~cm}^{-2}$

Table 1 Estimates of the diffusion constant $D$ at three different locations inside the well and at varying density, together with the mean values $\pm \mathrm{SD}$

\begin{tabular}{llllll}
\hline Cells/scm & 200 & 3,000 & 10,000 & 15,000 & 20,000 \\
\hline Coeff D1 & 0.31 & 0.30 & 0.37 & 0.31 & 0.31 \\
Coeff D2 & 0.18 & 0.23 & 0.32 & 0.30 & 0.26 \\
Coeff D3 & 0.23 & & 0.40 & 0.34 & 0.30 \\
Mean \pm SD & $0.24 \pm 0.07$ & $0.26 \pm 0.04$ & $0.36 \pm 0.04$ & $0.31 \pm 0.02$ & $0.30 \pm 0.03$ \\
\hline
\end{tabular}

\subsection{Image analysis of alignment}

Standard image segmentation algorithms proved unable to distinguish between individual cells and the background. Thus to identify patches of alignment and estimate their size quantitatively, both in the numerical simulations and in the experiment, we proceed in two steps. First, we apply a special class of filters to images such as Fig. 1 that reveal the dominant local axis of orientation. Second, we estimate the average size of cell clusters with a common orientation through a discrete statistical measure, which is then used to compare numerical simulations with experiments.

To classify cells according to their orientation, we opt for Gabor filters (Gabor 1946; Forsyth and Ponce 2003) which consist of a local Gaussian kernel of width $\sigma$, multiplied by a plane wave with distinct orientation $\theta$ and frequency $\omega$ :

$$
\begin{aligned}
G\left(x^{\prime}, y^{\prime}\right) & =\exp \left\{-\frac{1}{2}\left(\left(\frac{x^{\prime}}{\sigma}\right)^{2}+\left(\frac{y^{\prime}}{\sigma}\right)^{2}\right)\right\} \cos \left(2 \pi \omega x^{\prime}\right) \\
x^{\prime} & =x \cos (\theta)+y \sin (\theta), \quad y^{\prime}=y \cos (\theta)-x \sin (\theta),
\end{aligned}
$$



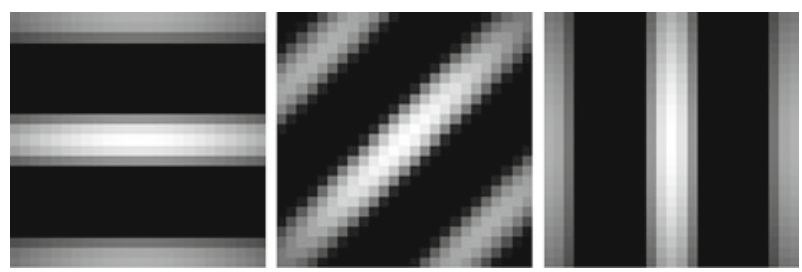

Fig. 3 Two-dimensional Gabor filters with frequency $\omega=0.08$, scale $\sigma=12$, and orientations $\theta=$ $0, \pi / 4, \pi / 2$

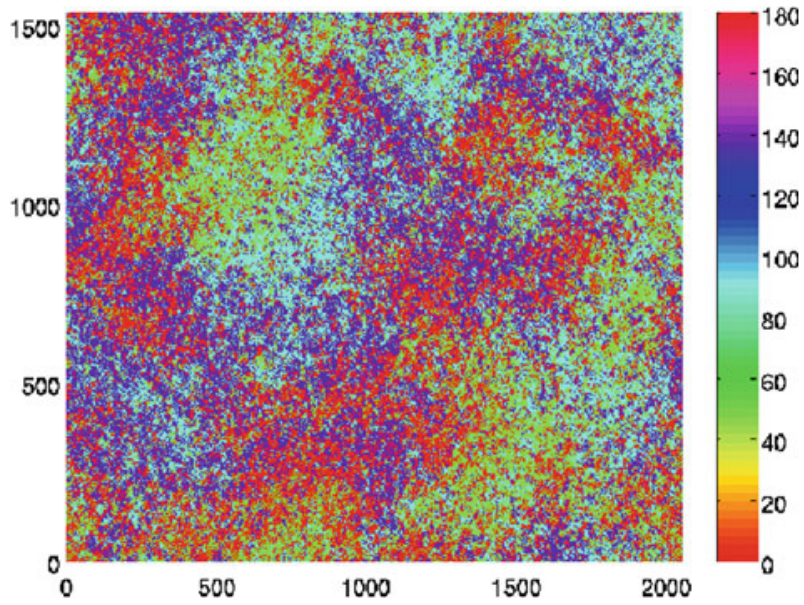

Fig. 4 The effect of Gabor filtering when applied to Fig. 1. The color indicates the dominant local direction of alignment (color figure online)

where unit length in $x$ (or $y$ ) corresponds to a single pixel. The typical width $\sigma=12$ and frequency $\omega=0.08$ for an array of aligned cells were determined a priori and remained fixed in all further analysis (see Fig. 3). Hence Gabor filters locally respond to patterns with spatial frequency $\omega$ and orientation $\theta$, within a subregion of size $\sigma$. Their two-dimensional extension is commonly used in image analysis and computer vision; they were also proposed as a model for the spatial summation properties of simple cells in the visual cortex (Daugman 1985).

To any image we apply a suite of Gabor filters for varying orientation at $45^{\circ}$ intervals and assign to each pixel location $(i, j)$ a distinct color $c_{i j}$ that corresponds to the highest filter response. Hence $c_{i j}$ reflects the dominant orientation at location $(i, j)$, and cells aligned with that particular orientation are thus revealed, as shown in Fig. 4.

Next, we estimate the typical cluster in a filtered image, such as in Fig. 4, either from experiment or numerical simulation. To do so, we assign to each pixel $(i, j)$ the value $p_{i j}(s)=1$ if it belongs to a cluster of size $s$, that is if at least $50 \%$ of the points within distance $s$ are of the same color; else, we set $p_{i j}(s)$ to zero. Summation over all pixels yields an estimate $r(s)$ of the number of pixels belonging to a cluster of size $s$ as 

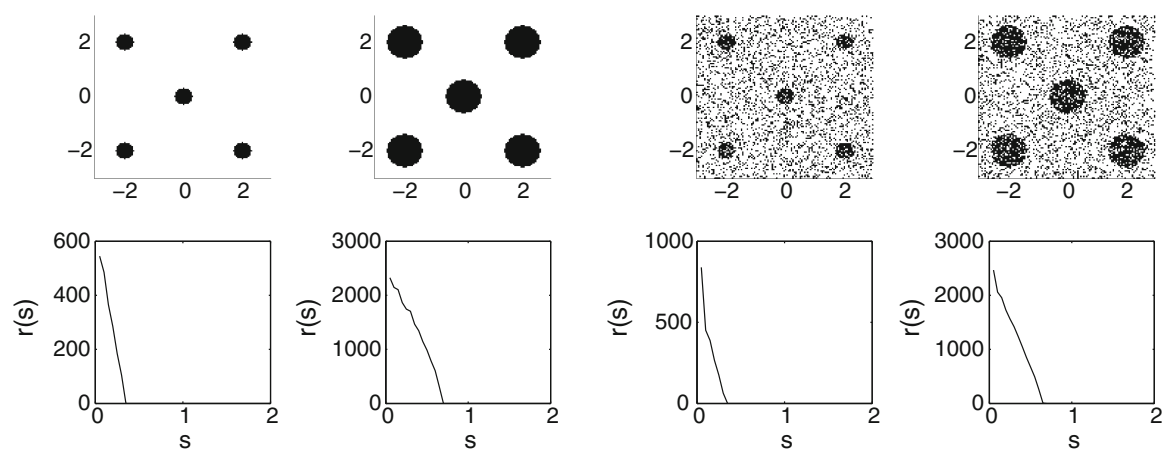

Fig. 5 Estimate of cluster size from two-dimensional synthetic texture images (top left), with corresponding cluster size function $r(s)$ below, as defined in (2.1) (bottom left). The intersection of $r(s)$ with the $s$-axis yields a robust estimate of the cluster size, even in the presence of added random noise (right)

$$
r(s)=\sum_{i j} p_{i j}(s), \quad p_{i j}(s)=\left\{\begin{array}{l}
1 \\
0
\end{array} .\right.
$$

The intersection of $r(s)$ with the $s$-axis yields a reliable estimate for the typical patch size, i.e. the largest cluster size, as illustrated with synthetic black and white data in Fig. 5. Moreover, comparison of the left and right frames in Fig. 5 demonstrates that the intersection of $r(s)$ with the $s$-axis is rather insensitive to added random noise.

\section{Mathematical model}

\subsection{Formulation}

Starting from the pioneering works of Edelstein-Keshet and Ermentrout (1990) and Mogilner and Edelstein-Keshet $(1995,1996)$, we now build a continuum model to describe the time evolution of a cell population of density $C(\theta, \mathbf{x}, t)$ in angle $\theta$ and two-dimensional space $\mathbf{x}=(x, y)$ at time $t$. During initial times the cells are essentially free to move in space and also turn their axis of orientation at random, similarly to fibroblasts. As the population density increases, however, cells come into contact. In Elsdale and Bard (1972), Edelstein-Keshet and Ermentrout (1990) and Erickson (1978) the underlying mechanism responsible for the directional motion and the resulting pattern formation is explained solely on the basis of single cell contact events for the case of related fibroblast cell cultures. This mechanism is a form of contact inhibition that cells experience when their lamellipodium touch. Indeed whenever mutual contact occurs within a small angle and hence only a portion of the lamellipodium touches, the cells alter their orientation accordingly and align, as observed by Elsdale and Bard (1972).

Following Mogilner and Edelstein-Keshet (1996) we now let $W\left(\mathbf{x}-\mathbf{x}^{\prime}, \theta-\theta^{\prime}\right)$ denote the rate at which a cell at $\mathbf{x}^{\prime}$ and $\theta^{\prime}$ moves to $\mathbf{x}$ and rotates to $\theta$ due to the impact of any surrounding cells. The angular velocity associated with this motion is then given by the gradient of $W$ at angle $\theta$ and position $\mathbf{x}$, due to the cumulative interaction 

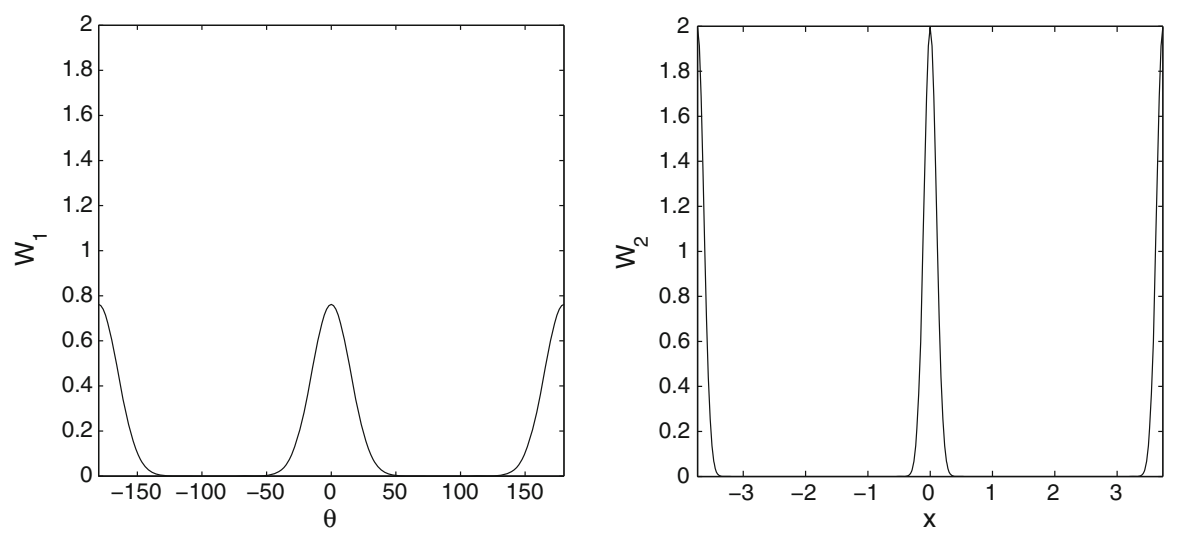

Fig. 6 The angular and spatial cellular interaction kernels from (3.3), (3.4): $W_{1}(\theta)$ with critical angle $\alpha=50^{\circ}($ left $)$, and $W_{2}(x)($ right $)$

with all other cells:

$$
\frac{\partial}{\partial \theta}(W \star C)(\mathbf{x}, \theta, t):=\frac{\partial}{\partial \theta} \int W\left(\mathbf{x}-\mathbf{x}^{\prime}, \theta-\theta^{\prime}\right) C\left(\theta^{\prime}, \mathbf{x}^{\prime}, t\right) d \theta^{\prime} d \mathbf{x}^{\prime}
$$

The gradient of the associated flux $C \partial_{\theta}(W \star C)$ then induces convective motion towards locations of higher concentration which corresponds to aggregation in space and alignment in angle; both compete with the inherent tendency of cells for random motion modelled by diffusive terms.

Next, the probabilities to align or to aggregate are assumed independent of each other, that is

$$
W\left(\mathbf{x}-\mathbf{x}^{\prime}, \theta-\theta^{\prime}\right)=W_{1}\left(\theta-\theta^{\prime}\right) W_{2}\left(\mathbf{x}-\mathbf{x}^{\prime}\right) .
$$

Moreover, experiments suggest that the probability of alignment $W_{1}$ decreases as the relative angle between neighboring cells increases (Elsdale and Bard 1972), whereas beyond a critical angle $\alpha$ cells no longer align; hence, $W_{1}$ must be positive and nonincreasing for $0 \leq \theta \leq \alpha$ but become negligible for $\alpha<\theta \leq \pi$. Since clockwise and anticlockwise turns are equally probable, $W_{1}$ must also be even. For simplicity, we assume that $W_{1}$ is Gaussian with mean zero and standard deviation $\alpha / 2$; other choices are possible and discussed in Edelstein-Keshet and Ermentrout (1990). After normalization, we thus obtain (Fig. 6)

$$
W_{1}(\theta)=\frac{1}{\alpha \sqrt{2 \pi}} e^{-\frac{2 \theta^{2}}{\alpha^{2}}}
$$

Since the strength of cell-to-cell interactions decreases with growing distance (Edelstein-Keshet and Ermentrout 1990), we again choose a Gaussian kernel for $W_{2}$, 


$$
W_{2}(\mathbf{x})=\frac{1}{2 \sigma^{2} \pi} e^{-\frac{|\mathbf{x}|^{2}}{2 \sigma^{2}}}, \quad \mathbf{x} \in\left[-L_{x}, L_{x}\right] \times\left[-L_{y}, L_{y}\right],
$$

where $L_{x}$ and $L_{y}$ denotes the size of the domain.

Our previous experiments indicate that the growth rate slows down, as the cell density increases locally in space, and that it eventually vanishes when the carrying capacity is reached because of limited space (Barbero et al. 2005). Therefore we model cell growth by a logistic term with growth rate $\rho$, where the growth rate reduction is determined by the population density at $x$ and $t$, that is by the marginal probability density $\int_{-\pi}^{\pi} C(t, x, y, \theta) d \theta$. The full logistic model, including the divergence of the drift of the cell population and random motion, previously derived in Mogilner and Edelstein-Keshet (1996), can then be written as

$$
\begin{aligned}
\frac{\partial C}{\partial t}= & \epsilon_{1} \frac{\partial^{2} C}{\partial \theta^{2}}+\epsilon_{2}\left(\frac{\partial^{2} C}{\partial x^{2}}+\frac{\partial^{2} C}{\partial y^{2}}\right) \\
& -\gamma \frac{\partial}{\partial \theta}\left(C\left[\frac{\partial W}{\partial \theta} * C\right]\right)-\gamma\left\{\frac{\partial}{\partial x}\left(C\left[\frac{\partial W}{\partial x} * C\right]\right)+\frac{\partial}{\partial y}\left(C\left[\frac{\partial W}{\partial y} * C\right]\right)\right\} \\
& +\rho C\left(1-\frac{L_{x} L_{y}}{K} \int_{-\pi}^{\pi} C(t, x, \theta) d \theta\right)
\end{aligned}
$$

where $\epsilon_{1}, \epsilon_{2}$ and $\gamma$ denote the diffusion and drift coefficient, respectively. In addition we note that upon using the definition (3.1) and functional forms (3.2)-(3.4), integration by parts yields the above drift terms.

\section{Stability}

\section{Linear stability analysis}

Before investigating numerically the emergence of coherent patterns in the full nonlinear model (3.5), it is instructive to investigate the stability properties of the homogeneous state, i.e. the state, where the density of cells in angular and spatial space is equally distributed. Linear stability analysis characterizes the effect of small perturbations on the early time evolution in angular and spatial space. We therefore expect good agreement with the early stages of the numerical solution of the fully nonlinear model (3.5).

If $\rho=0$, the homogeneous state $C \equiv \bar{C}$ is an exact solution of (3.5), since we then have $W \star C=C$. In this case, linearization of (3.5) about $C \equiv \bar{C}$ using the ansatz

$$
C(\mathbf{x}, \theta, t)=\bar{C}+\delta C_{n, q}^{\prime}(\mathbf{x}, \theta, t),
$$

where the amplitude of the perturbation $\delta \ll 1$ is small, results in an eigenvalue problem for the integro-differential operator previously analyzed in Edelstein-Keshet and Ermentrout (1990) and Mogilner and Edelstein-Keshet (1996). In particular, 
Mogilner and Edelstein-Keshet (1996) showed for an unbounded spatial domain that the functions

$$
u_{q_{1}}(x)=e^{i q_{1} x}, \quad u_{q_{2}}(y)=e^{i q_{2} y}, \quad z_{n}(\theta)=e^{i n \theta}, \quad q_{1}, q_{2} \in \mathbb{R}
$$

form a complete set of orthogonal eigenfunctions for the spatial and angular Laplace operator with eigenvalues $q_{1}, q_{2}$ for the spatial and $n=0,1,2, \ldots$ for the angular diffusion operator, respectively. In addition, they proved that (4.2) are not only the eigenfunctions of the Laplace operators, but also of the convolution operators $W_{1} *$ and $W_{2} *$, where the eigenvalues are the Fourier coefficients denoted by $\hat{W}_{n}$ and $\hat{W}_{q}=\hat{W}_{q_{1}} \hat{W}_{q_{2}}$, where $q=\sqrt{\left(q_{1}^{2}+q_{2}^{2}\right)}$ and

$$
\hat{W}_{n}=\frac{1}{\pi} \int_{-\pi}^{\pi} W_{1}(\theta) e^{-i n \theta} d \theta, \quad \hat{W}_{q_{j}}=\int_{0}^{L_{x_{j}}} W_{2}\left(x_{j}\right) e^{-i q_{j} x_{j}} d x_{j},
$$

where $j=1,2$ and $x_{1}=x, x_{2}=y$. From the normal modes ansatz

$$
C_{n, q}^{\prime}(\mathbf{x}, \theta, t)=e^{\lambda t} u_{q_{1}}(x) u_{q_{2}}(y) z_{n}(\theta)
$$

the stability of the homogeneous state is then found from the solution of the equation

$$
\lambda=-r+\bar{C} s
$$

where

$$
r=\left(\epsilon_{1} n^{2}+\epsilon_{2} q^{2}\right) \text { and } s=\hat{W}_{q} \hat{W}_{n} \gamma\left(n^{2}+q^{2}\right)
$$

for all $q_{1}, q_{2}$ and $n$. Hence the condition for instability of the homogeneous solution is given by $\lambda>0$. Thus, any increase in the diffusion coefficients $\epsilon_{1}, \epsilon_{2}$ tends to stabilize the system, while the cell-to-cell interaction terms $\hat{W}_{n}$ and $\hat{W}_{q}$ tend to destabilize the system, for increasing values of $n, q$, unless $W_{n}$ is zero. Moreover, for any particular values of $\epsilon_{1}, \epsilon_{2}, n, q$, the constant state $\bar{C}$ becomes unstable at sufficiently high cell density, unless $\hat{W}_{n}$ or $\hat{W}_{q}$ vanishes.

For our extended model with logistic growth, where $\rho \neq 0$, the homogeneous state about which we linearize is now time-dependent, due to the slow mass increase. Thus we make the ansatz

$$
C(\theta, \mathbf{x}, t)=\bar{C}(t)+\delta C_{n, q}^{\prime}(\theta, \mathbf{x}, t)
$$

with $C_{n, q}^{\prime}=\hat{C}_{n, q}(t) e^{i(q x+n \theta)}$, since now the standard normal modes ansatz may lead to non-normal linear systems with non-orthogonal eigenfunctions (see Trefethen et al. 1993), for instance, for a more detailed discussion of such problems in the context 
of hydrodynamics. Our slightly more general ansatz for $C(\theta, \mathbf{x}, t)$ then leads to the following differential equation for $\hat{C}_{n, q}$

$\frac{d \hat{C}_{n, q}}{d t}=\left[-\left(\epsilon_{1} n^{2}+\epsilon_{2} q^{2}\right)+\bar{C}(t) \hat{W}_{q} \hat{W}_{n} \gamma\left(n^{2}+q^{2}\right)+\rho\left(1-\frac{\bar{C}(t)}{\kappa}\right)\right] \hat{C}_{n, q}(t)$

where

$$
\bar{C}(t)=\frac{\kappa}{1+C_{1} \kappa e^{-\rho t}}
$$

is the solution of the leading order problem and represents the slowly growing mass until the carrying capacity is reached. The constant $C_{1}=1 / \bar{C}(0)-1 / \kappa$, where $\bar{C}(0)$ is chosen to be the same as $\bar{C}$ in the original problem and we denote $\kappa=K /\left(2 \pi L_{x} L_{y}\right)$. Hence, the growth rate is given here by

$$
\ln \left(\hat{C}_{n, q}(t)\right)=(-r+s \kappa) t+\frac{s \kappa-\rho}{\rho} \ln \left(1+C_{1} \kappa e^{-\rho t}\right)+\text { const }
$$

We note that now the additional parameter $K$, the carrying capacity, will have a decisive impact on the stability properties of the solutions.

\section{Comparison of the full model with linear stability}

To compare the results from linear stability analysis to those from the numerical simulation of the full problem, we choose as an example the simple case for which $\rho=0$ and $\gamma=1$. We set the (constant) base state $\bar{C}=25$, let $\epsilon_{1}=0.0025$ and $\epsilon_{2}=0.5$ so that for $n>0$ and $q=0$ the base state is unstable according to linear stability analysis. Now, we determine $n_{\max }$ such that the growth rate is maximal, i.e. $\sigma_{\max }=\sigma\left(n_{\max }, q_{\max }\right)$ (here $\left.q=q_{\max }=0\right)$. Thus, we can find $n_{\max }$ which is at most $O(1)$ with a $\sigma_{\max }$ not too small, together with the corresponding eigenfunction $C_{n, q}^{\prime}$ and a corresponding asymptotic growth rate $\lambda$.

Next, we initialize our nonlinear simulation with the initial data

$$
\bar{C}+\delta C_{n, q}^{\prime}, \quad \text { such that } \delta \leq \min \left(0.1,0.1 \frac{\sigma_{\max }}{n_{\max }^{2}}\right)
$$

to ensure that the correction term does not invalidate the original assumptions of linear stability analysis.

In Fig. 7, $\log \|C\|$ with

$$
\|C\|=\frac{\max _{\theta, x, y}|C(\theta, x, y, t)-\bar{C}|}{\delta}
$$

is shown versus $t$, both for the solution of the fully nonlinear model and for that from linear stability. Note that the growth rate of the linearized problem for the extended 

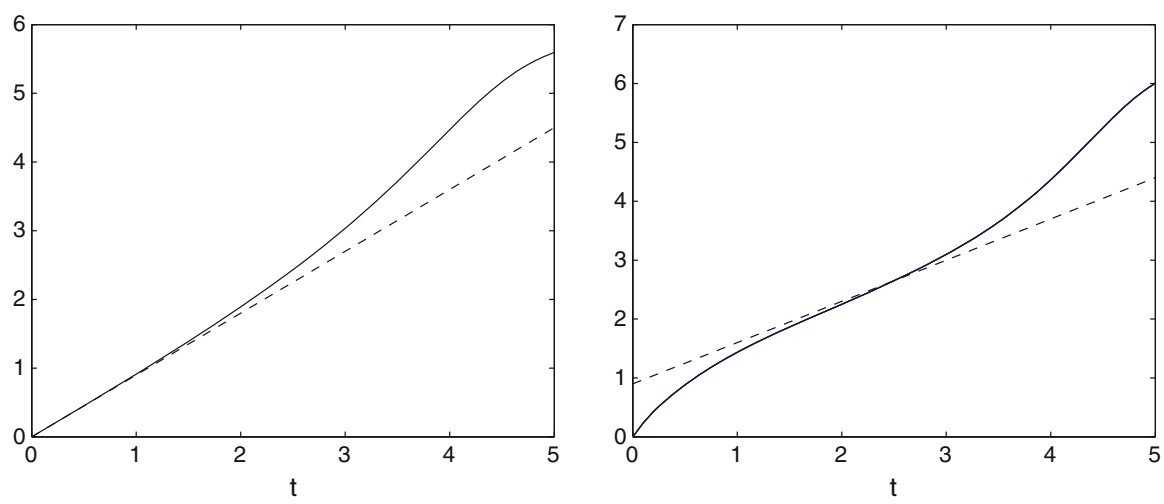

Fig. 7 Comparison of the growth rates for the fully nonlinear and the linearized models. Left $\rho=0$ : the dashed line is $\lambda t$, where $\lambda$ is given by the solution of the linear stability problem (4.5). The solid curve denotes $\ln (\| C||)$. Right, $\rho=0.2$ : the dashed line shows the long-time behaviour of the solution of (4.10). The solid curve results from the solution to the full problem (3.5)

model, i.e. where $\rho \neq 0$, now also depends on time. Once initial transients have died out, both models agree, as expected. As time progresses, however, the dynamics of the full model deviate from those of the linearized problem. Thus, the evolving patterns may deviate from those predicted by linear stability theory, in particular at later times, as the cell culture reaches confluence, depending on parameter values.

In Fig. 7 we show a comparison of the growth rates for the fully nonlinear and the linearized models, with the above set of parameters. This example illustrates the following generic behaviour. For $\rho=0$, we observe agreement right from the beginning, since the perturbation corresponds to an exact eigenfunctions, as in the linear stability problem. For the extended model with $\rho=0.2$ and $K=1,220$, for instance, we observe that the long-time behaviour of the solution of (4.10) compares well with the solution to the full problem (3.5). Eventually though, the nonlinear terms come into play and the solution of the full model deviates from the prediction of the linear model.

\section{Comparison of simulations with experiments}

\subsection{Parameter values}

To compare computational results from any mathematical model with those from experiment, it is crucial to have accurate estimates of the parameter values. While the values of most parameters were determined quite accurately from experiment, uncertainties about some of them remained.

In particular, the estimation of the spatial diffusion coefficient $\epsilon_{2}$ was performed by hand following the positions of the moving cells. From the experiment (Table 1), the average spatial diffusion coefficient $\epsilon_{2}=0.29 \mu \mathrm{m}^{2} / \mathrm{s}=0.025 \mathrm{~mm}^{2} /$ days. Because cells do not change their orientation in a continuous way, $\epsilon_{1}$ could not be determined from those measurements, mainly because cells also contract, become almost 

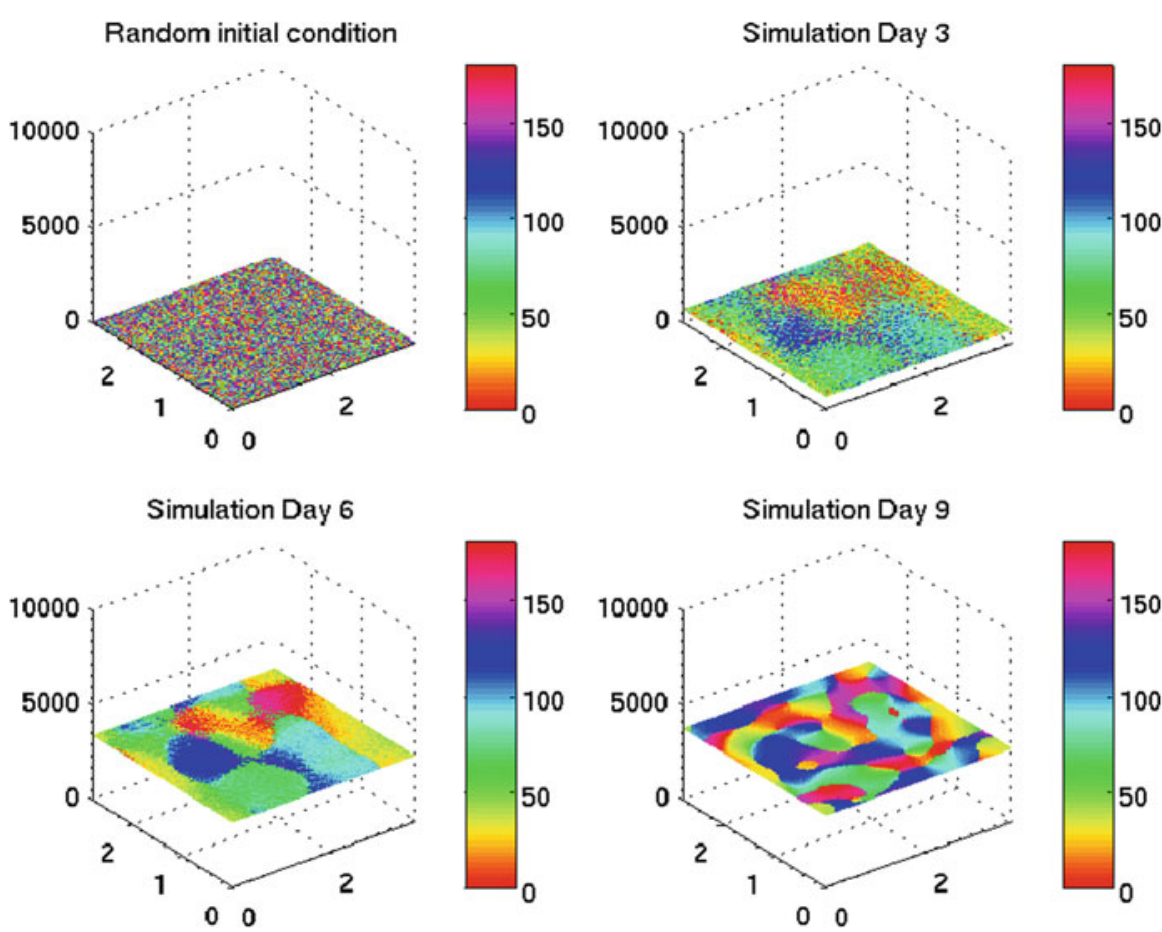

Fig. 8 Snapshots of the cell density at different times. At each point $(x, y)$ in the computational domain, the marginal angular density of $C$ is shown, that is the number of cells at position $(x, y)$ and time $t$; the color represents the angle for which $C(x, y, \theta, t)$ is maximal (color figure online)

spherical, and elongate randomly, excluding the determination of a well-defined axis of orientation. In our numerical simulations, we chose values for the angular diffusion coefficient $\epsilon_{1}$, which is the mean square displacement in angular space per day, to range from 0.025 (Figs. 8, 9, 11) to 0.0025 (Fig. 10). The values for $\rho$ and $K$ are always given by 1.2 and 4,000 cells $/ \mathrm{mm}^{2}$ and have been determined previously in Barbero et al. (2005). They were used as initial guess for a nonlinear leastsquares parameter fit to the time evolution of the total mass. The size of the domain $L_{x}=3.75 \mathrm{~mm}$ and $L_{y}=2.75 \mathrm{~mm}$ was chosen to match the area observable under the microscope. In Elsdale and Bard (1972), the critical angle $\alpha$ was obtained for fibroblast cultures by inspection of relative angles between cells at confluence. Because of the strong similarity between cytokine cultured chondrocytes and fibroblasts, we used the same value here, i.e. $\alpha=20^{\circ}$ for our simulations, except in Fig. 11, where we also include results for critical angles $\alpha=40^{\circ}$ and $60^{\circ}$. Since chondrocytes only attach when they are very close to each other, we chose the standard deviation $\sigma=0.01 \mathrm{~mm}$ for the spatial interaction kernel to be about the length of a single cell. The value of $\gamma=0.0005$ essentially sets the convective time scale and was obtained by fitting the cluster size from the simulation to that obtained from experiment (see Sect. 2.3). 

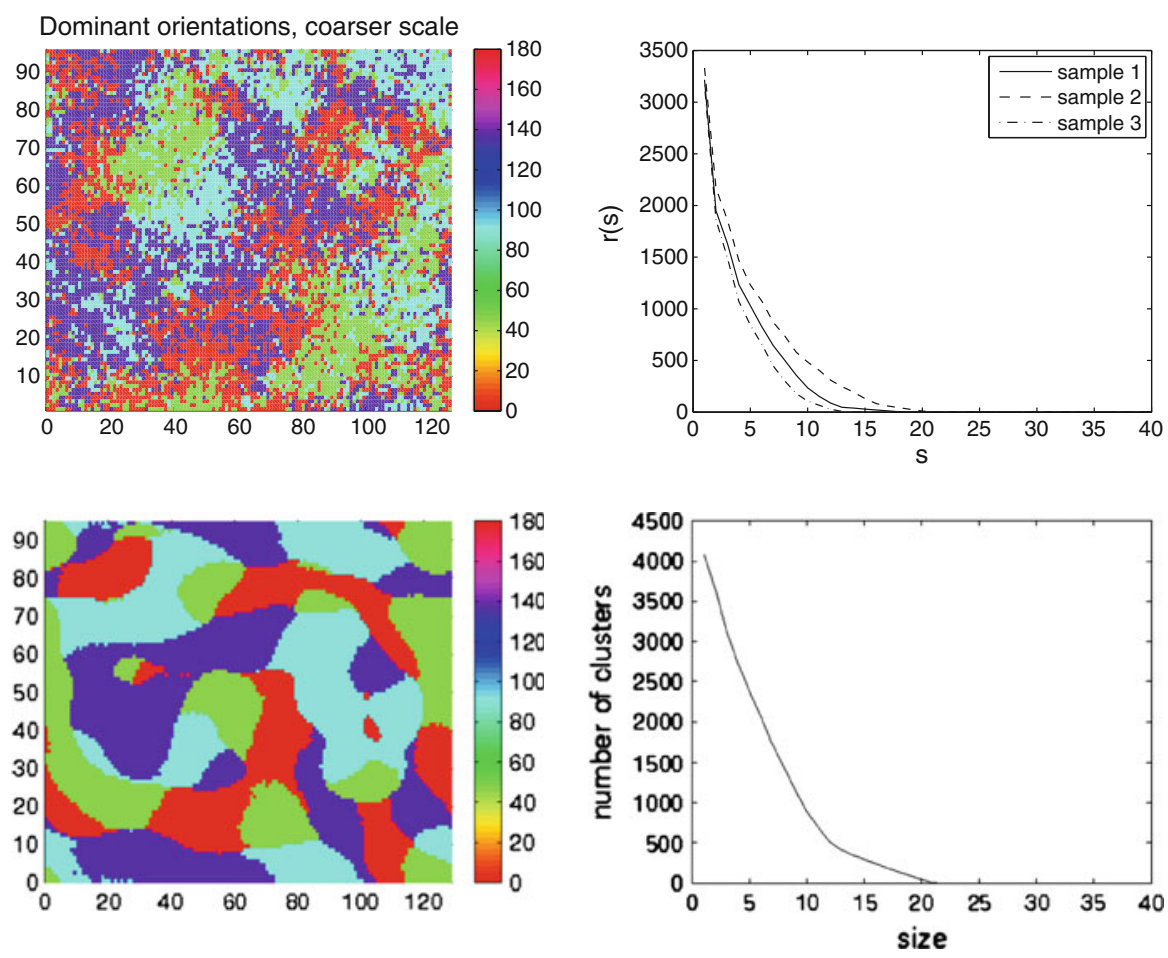

Fig. 9 Comparison of simulation (bottom) with experiment (top). Here color indicates the local dominant orientation of the cells. The cluster size for three samples from the same donor (top) and for the simulation (bottom) are shown on the right (color figure online)
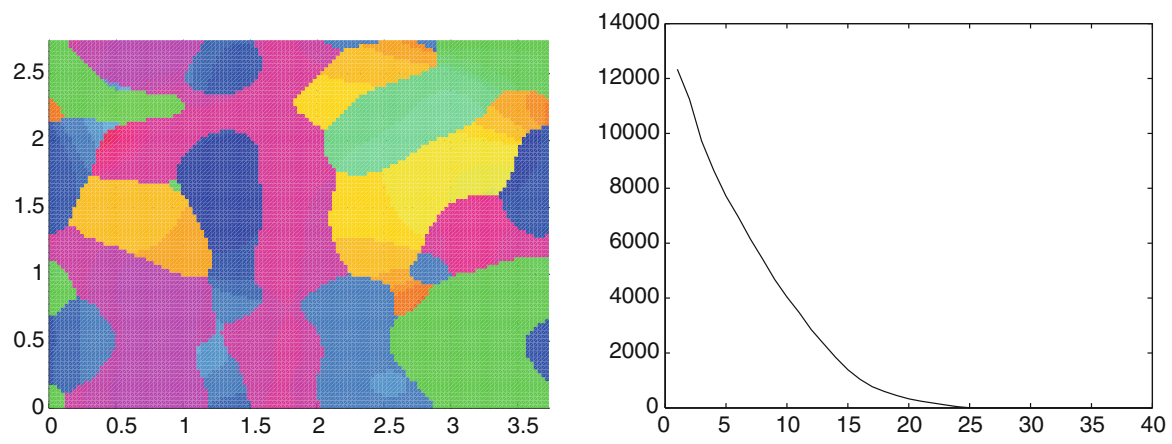

Fig. 10 The cell density is shown at confluence for the smaller angular diffusion coefficient: $\epsilon_{1}=0.0025$. Left the dominant cell orientation; right the cluster size function $r(s)$

\subsection{Numerical simulations}

Starting from a random initial distribution at $t=0$, we solve (3.5) using the numerical method described in Appendix A and the parameter values listed above. In Fig. 8, snapshots of the cell density at different times are shown. Here at each point $(x, y) \in \Omega$ 

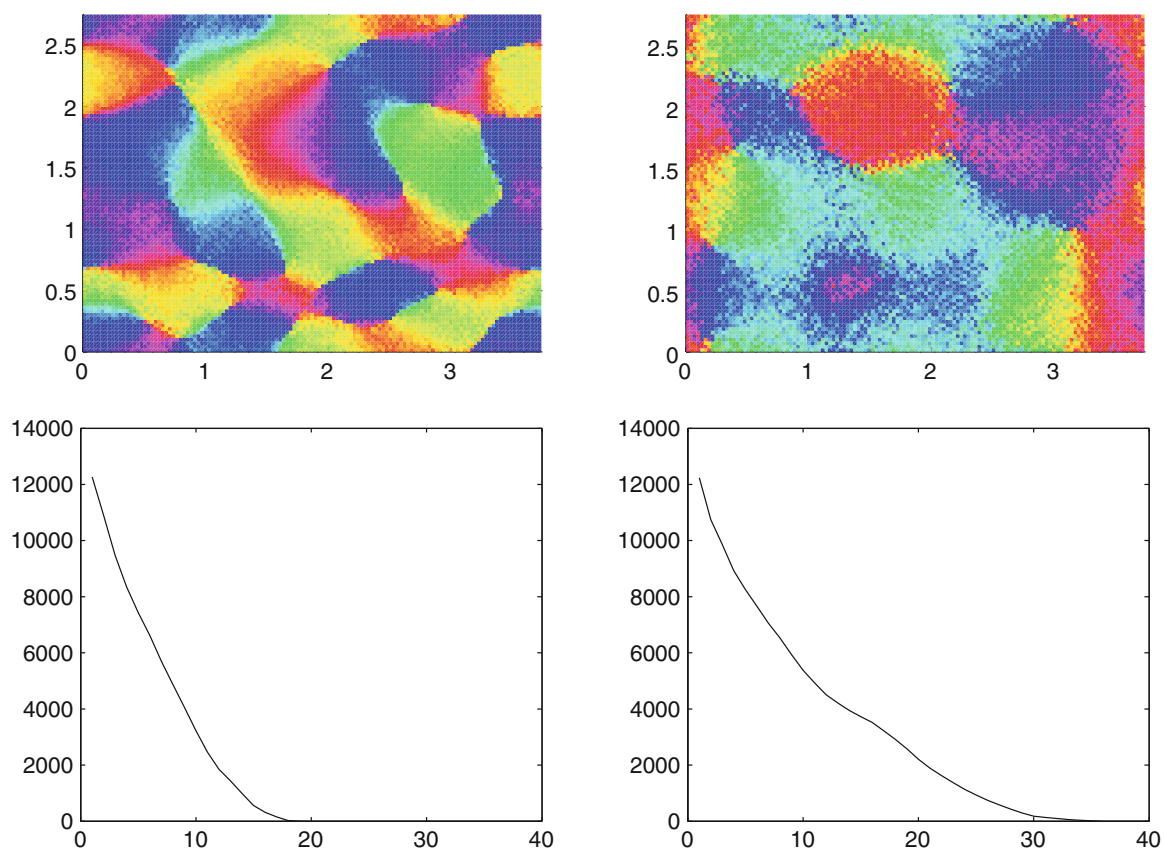

Fig. 11 The cell density is shown at confluence for larger critical angles: $\alpha=40^{\circ}$ (left) and $\alpha=60^{\circ}$ (right). The corresponding cluster size functions $r(s)$ are shown below

the marginal spatial cell density of $C$, that is the integral of $C(x, y, \theta, t)$ over $\theta$, is displayed. The color used at any point $(x, y)$ corresponds to the angle, where $C(x, y, \theta, t)$ is maximal; hence, it represents the local dominant orientation of the cells. We observe that the number of cells increases uniformly throughout the computational domain $\Omega$, yet past day 6 several patches of cells with a common orientation emerge and settle in a stationary configuration by day 9 ; note that the total number of cells hardly changes beyond day 6 anymore.

In Fig. 9 we compare the simulation with the experimental data using Gabor filters for post-processing both (see Sect. 2.3). In doing so the spatial resolution of the microscope image was coarsened to match that of the simulation, while the angular dependence over $[0, \pi)$ was divided into four classes, that is sub-intervals of identical lengths, each one assigned with a different color. The cluster size (intersection of $r(s)$ with the $x$-axis, see Sect. 2.3) was calculated for three samples from the same donor. By fitting the average cluster size from the simulation to that from experiment, between 15 and 20 pixels or about $0.5 \mathrm{~mm}$, we determined the standard value of $\gamma$, as shown in Fig. 9.

Once the model has been validated through comparison to experiment, it is instructive to change the value of individual parameters to study their effect on the size and shape of the patterns at confluence. Thus we can also evaluate the parameter sensitivity of the model and address the uncertainties associated with some of the values obtained from experiment. For instance, the reduction of the angular diffusion coefficient $\epsilon_{1}$ 
has little effect on the size of the patterns, but the interfaces appear more well-defined in contrast to the standard case: compare Figs. 9 and 10. An increase in the critical angle $\alpha$ instead, results in larger and increasingly irregular patterns, while the uniform spatial population density is maintained, as shown in Fig. 11.

\section{Concluding remarks}

Starting from the classical models by Mogilner and Edelstein-Keshet (1996), we have developed a mathematical model for proliferating chondrocytes, cultured with specific growth factors, by including logistic growth and studied the patterns emerging at confluence through experiments and simulation. Most parameters in the model were obtained directly through independent experiments or from our previous micro-colony tests (Barbero et al. 2005). Guided by these parameter studies we arrived at reasonable parameter values for comparison to the experimentally observed cell patterns at confluence. Linear stability analysis was used as guidance through the range of unstable parameter values, as their interplay leads to pattern formation; their improved understanding and control will be useful in the future design of engineered tissue.

For the time integration of the nonlinear integro-partial differential equation, we opted for Runge-Kutta-Chebyshev methods which permit much larger time steps than standard Runge-Kutta methods while nonetheless remaining fully explicit. Quantitative comparison of experimental data with the numerical simulations was achieved in two steps. First, we visualized the orientation and alignment of the cells with Gabor filters. Then, we determined the average cluster size of the cell population both in the simulation and the experiment.

From the stability analysis and the simulations, we were able to determine key parameters for pattern formation. In particular, we find that the total number of dominant directions of alignment in a cell culture is mainly regulated by the critical angle, below which the probability that cells align is high. Indeed, smaller values in the critical angle $\alpha$ for cell-cell interactions lead to arrays of aligned cells, as observed in experiments, whereas larger values lead to a single dominant direction of alignment. Regarding the diffusion and drift coefficients, both tend to destabilize the homogeneous state and thereby lead to pattern formation. For fixed diffusion coefficients, the average pattern size typically scales with the drift coefficient, $\gamma$, although the number of dominant directions of alignment remains identical, as it is regulated by the critical angle.

While we think that continuum models in combination with some local experimental analysis yields convincing evidence to capture the large scale long-time structures of proliferating cell cultures, our work also leaves a number of open tasks and questions. Apart from the study of aggregation patterns, which has been left open, the experimental determination of the remaining parameters, in particular drift parameters but also angular diffusion coefficients will be an important future task. We believe that in principle more sophisticated image analysis and segmentation software would allow the automatic tracking of larger number of cells and yield both more refined and improved statistics. Through a new set of experiments, more experimental studies such as those by Elsdale (1973) are needed in order to establish more accurately the 
critical angle for cell alignment for the particular cells under consideration without relying on similar cases from the literature.

Acknowledgments We thank Assyr Abdulle, Andreas Münch, Ben Schweizer, and Thomas Vetter for useful comments and suggestions.

\section{Appendix A: Numerical methods}

Here we describe the numerical discretization in space and time for solution of (3.5). We restrict the computations to a small subregion $\Omega$ inside the experimental well. Thus, boundary effects due to the finite size of the well are negligible and we may impose periodic boundary conditions at the boundary of the computational domain $\Omega=\left[0, L_{x}\right] \times\left[0, L_{y}\right]$. For the numerical approximation of (3.5) all spatial derivatives are approximated by second-order centered finite differences on a regular grid. The convolution integrals are computed by trapezoidal quadrature, which yields exponential convergence for periodic analytic functions (Kress 1999). Hence the numerical discretization error is second-order accurate in space and angle.

For parabolic problems standard explicit Runge-Kutta schemes impose rather stringent restrictions on the time-step for numerical stability, typically $\Delta t \leq C \Delta x^{2}$, and hence are notoriously inefficient (Hairer and Nørsett 1987). In contrast, implicit methods waive those time-step restrictions but would require here the solution of a nonlinear integro-differential boundary value problem at every time step, a rather high price to pay.

To avoid the above mentioned difficulties, we opt for Runge-Kutta-Chebyshev methods instead, which are fully explicit while allowing larger time-steps. Instead of maximizing the accuracy, RK-Chebyshev methods maximize the interval $[-\ell, 0]$ of the negative real axis contained in the stability region (Hairer and Nørsett 1987; Markoff 1916). Because $\ell$ is proportional to $s^{2}$, for a fixed number of stages, $s$, any reduction of the mesh size $\Delta x$ can be counterbalanced by an equivalent increase of the number of stages while keeping the time-step $\Delta t$ fixed. Therefore $\mathrm{RK}-\mathrm{Chebyshev}$ methods circumvent the crippling quadratic increase in the number of time-steps of traditional RK methods that results from any linear reduction of the mesh size (Franklin 1959; Gouillou and Lago 1960; Yuan 1958).

For instance, the first-order $s$-stage RK-Chebyshev method for the initial-value problem

$$
y^{\prime}(t)=f(y), \quad y(0)=y_{0},
$$

is given by

$$
\begin{aligned}
& g_{0}=y_{0}, \\
& g_{1}=y_{0}+\left(1 / s^{2}\right) \Delta t f\left(g_{0}\right), \\
& g_{i}=\left(2 / s^{2}\right) \Delta t f\left(g_{i-1}\right)+2 g_{i-1}-g_{i-2}, \\
& y_{1}=g_{s} .
\end{aligned}
$$



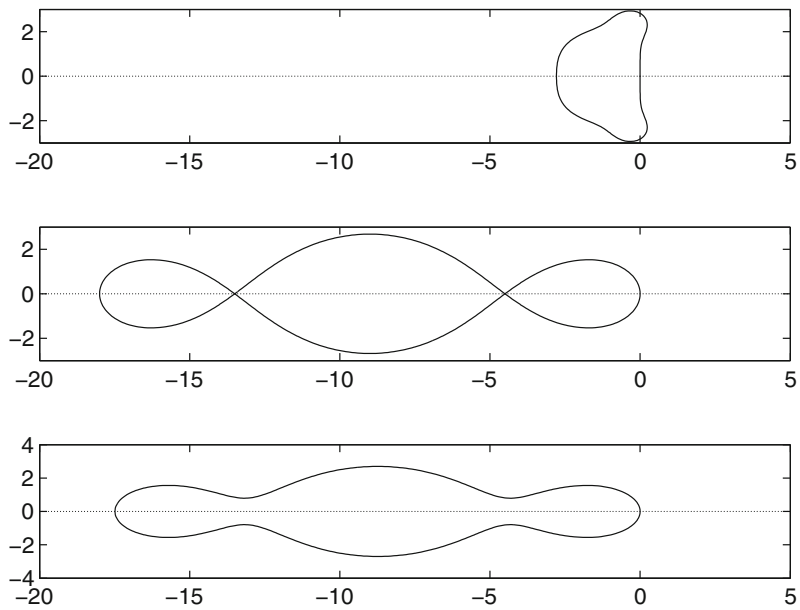

Fig. A.1 Stability regions: fourth-order RK4 (top) and first-order 3-stage Chebyshev methods without damping (middle) and with damping (bottom). The stability regions of the RK-Chebyshev method is about nine times larger then that of the standard RK4

In Fig. A.1 we observe that the stability regions of the 3-stage RK-Chebyshev method is about nine times larger than that of the standard fourth-order RK4. Following Hairer (2002), we eliminate the two intersections where the stability region shrinks to zero by adding small damping of size $\epsilon>0$. Let $\epsilon>0$ and $T_{S}(x)$ denote the Chebyshev polynomial of degree $s$ (Abramowitz 1964). Then the damped RK-Chebyshev method for (A.1) is given by

$$
\begin{aligned}
& g_{0}=y_{0} \\
& g_{1}=y_{0}+\Delta t\left(w_{1} / w_{0}\right) f\left(g_{0}\right), \\
& g_{i}=\frac{1}{T_{i}\left(w_{0}\right)}\left[2 w_{1} \Delta t T_{i-1}\left(w_{0}\right) f\left(g_{i-1}\right)+2 w_{0} T_{i-1}\left(w_{0}\right) g_{i-1}-T_{i-2}\left(w_{0}\right) g_{i-2}\right], \\
& y_{1}=g_{s} .
\end{aligned}
$$

where

$$
R_{s}(z)=\frac{1}{T_{s}\left(w_{0}\right)} T_{s}\left(w_{0}+w_{1} z\right), \quad w_{0}=1+\frac{\epsilon}{s^{2}}, \quad w_{1}=\frac{T_{s}\left(w_{0}\right)}{T_{s}^{\prime}\left(w_{0}\right)}
$$

As illustrated in Fig. A.1 for $\epsilon=0.05$, the stability domain is now slightly shorter (by a factor $4 \epsilon s^{2} / 3$ ), but its boundary remains at a safe distance form the real axis (Hairer 2002).

When the right-hand side in (A.1) explicitly depends on time, the terms involving $f\left(g_{i}\right)$ in (A.6)-(A.9) are replaced by $f\left(g_{i}, t_{i}\right)$. The precise times $t_{i} \in[0, \Delta t]$ where $f$ needs to be evaluated are determined by augmenting (A.1) with the trivial differential 
equation,

$$
z^{\prime}(t)=1, \quad z(0)=t_{0}
$$

and applying (A.6)-(A.9) to it. Thus for $t \in[0, \Delta t]$ we have

$$
\begin{aligned}
& t_{0}=0 \\
& t_{1}=\Delta t\left(w_{1} / w_{0}\right) \\
& \left.t_{i}=\frac{1}{T_{i}\left(w_{0}\right)}\left[2 w_{1} \Delta t T_{i-1}\left(w_{0}\right)\right)+2 w_{0} T_{i-1}\left(w_{0}\right) t_{i-1}-T_{i-2}\left(w_{0}\right) t_{i-2}\right],
\end{aligned}
$$

and so forth during subsequent time steps.

\section{References}

Abramowitz M, Stegun IA (1964) Handbook of mathematical functions with formulas, graphs, and mathematical tables. Dover Publications, New York

Barbero A, Grogan S, Schafer D, Heberer M, Mainil-Varlet P, Martin I (2004) Age related changes in human articular chondrocyte yield, proliferation and post-expansion chondrogenic capacity. Osteoarthr Cartil 12:476-484

Barbero A, Palumberi V, Wagner B, Sader R, Grote MJ, Martin I (2005) Experimental and mathematical study of the influence of growth factors on the growth kinetics of adult human articular chondrocytes. J Cell Physiol 204:830-838

Barbero A, Ploegert S, Heberer M, Martin I (2003) Plasticity of clonal populations of dedifferentiated adult human articular chondrocytes. Arthr Rheum 48:1315-1325

Beattie G, Cirulli V, Lopez A, Hayek A (1997) Ex vivo expansion of human pancreatic endocrine cells. J Clin Endocrinol Metab 82:1852-1856

Carpenter M, Cui X, Hu Z, Jackson J, Sherman S, Seiger A, Wahlberg L (1999) In vitro expansion of a multipotent population of human neural progenitor cells. Exp Neurol 158:265-278

Chipot M, Edelstein-Keshet L (1983) A mathematical theory of size distribution in tissue culture. J Math Biol 16:115-130

Civelecoglu G, Edelstein-Keshet L (1994) Modelling the dynamics of f-actin in the cell. Bull Math Biol $56: 587-616$

Daugman J (1985) Uncertainty relations for resolution in space, spatial frequency, and orientation optimized by two-dimensional visual cortical filters. J Opt Soc Am A 2:1160-1169

Deasy B, Qu-Peterson Z, Greenberger J, Huard J (2002) Mechanisms of muscle stem cell expansion with cytokines. Stem Cells 20:50-60

Deenick E, Gett A, Hodgkin P (2003) Stochastic model of T dell proliferation: a calculus revealing il-2 regulation of precursor frequencies, cell cycle time, and survival. J Immunol 170:4963-4972

Edelstein-Keshet L, Ermentrout G (1990) Models for contact-mediated pattern formation: cells that form parallel arrays. J Math Biol 29:3-58

Elsdale T (1973) The generation and maintenance of parallel arrays in cultures of diploid fibroblasts. In: Kulonen E, Pikkarainen J (eds) Biology of fibroblast. Academic Press, London, pp 41-58

Elsdale T, Bard J (1972) Collagen substrata for studies on cell behaviours. J Cell Biol 54:626-637

Erickson C (1978) Analysis of the formation of parallel arrays in bhk cells in vitro. Exp Cell Res 115: 303-315

Forsyth DA, Ponce J (2003) Computer vision, a modern approach. Prentice Hall, Englewood Cliffs

Franklin J (1959) Numerical stability in digital and anlogue computation for diffusion problems. J Math Phys 37:305-315

Gabor D (1946) Theory of comminication. J IEE 93(26):429-457

Gouillou A, Lago B (1960) Domaine de stabilité associé aux formules d'intégration numérique d'équations différentielles, à pas séparés et à pas liés. Recherche de formules à grand rayon de stabilité. Ier Congr. Ass. Fran. Calcul. AFCAL pp 43-56 
Hairer EWG (2002) Solving ordinary differential equation II. Springer, Berlin

Hairer E, Nørsett SPWG (1987) Solving ordinary differential equations. I: nonstiff problems. Springer, Berlin

Jakob M, Demarteau O, Schafer D, Hinterman B, Dick W, Heberer M, Martin I (2001) Specific growth factors during the expansion and redifferentiation of adult human articular chondrocytes enhance chondrogenesis and cartilaginous tissue formation in vitro. J Cell Biochem 81:368-377

Kress R (1999) Linear integral equations. Springer, Berlin

Langer R, Vacanti J (1993) Tissue engineering. Science 260:920-926

Markoff CL (1916) Ueber Polynome, die in einem gegebenen Intervall möglichst wenig von Null abweichen. Math Ann 77:213-258

Mogilner A, Edelstein-Keshet L (1995) Selecting a common direction, how orientational order can arise from simple contact responses between interacting cells. J Math Biol 33:619-660

Mogilner A, Edelstein-Keshet L (1996) Spatio-angular order in populations of self-aligning objects: formation of oriented patches. Physica D 89:346-367

Mogilner A, Edelstein-Keshet L, Ermentrout GB (1996) Selecting a common direction. II. peak-like solutions representing total alignment of cell clusters. J Math Biol 34:811-842

Sharma B, Elisseeff JH (2004) Engineering structurally organized cartilage and bone tissues. Ann Biomed Eng 32:148-159

Stewart J, Masi T, Cumming A, Molnar G, Wentworth B, Sampath K, McPherson J, Yaeger P (2003) Characterization of proliferating human skeletal muscle-derived cells in vitro: differential modulation of myoblast markers by tgf-beta2. J Cell Physiol 196:70-78

Trefethen L, Trefethen A, Reddy S, Driscoll D (1993) Hydrodynamics without eigenvalues. Science 261:578

Trinkaus JP (1985) Further thoughts on directional cell-movement during morphogenesis. J Neurosci Res 13:1-19

Yuan C (1958) Some difference schemes for the solution of the first boundary value problem for linear differential equations with partial derivatives. Master's thesis, Moscow State University 